\title{
LA CONVERSIONE DI ENERGIA SOLARE IN ENERGIA CHIMICA
}

\author{
ELENA SELLI $(*)$ \\ Nota presentata dal m.e. Stefano Maiorana \\ (Adunanza del 5 ottobre 2017)
}

SuNTO. - L'energia costituisce la questione più importante del ventunesimo secolo. A causa di gravi problemi ambientali, legati soprattutto all'inquinamento dell'aria nelle zone urbane e all'effetto serra con conseguente riscaldamento globale, è necessario sostituire i combustibili fossili con fonti di energia rinnovabili. Accanto all'energia eoli$\mathrm{ca}$, progressivamente sempre più utilizzata, l'energia solare rappresenta una forma di energia pulita, abbondante, diffusa ed economica, che può essere convertita in calore, elettricità o nei cosiddetti combustibili solari, utilizzando materiali in grado di assorbire la luce solare in modo efficiente e consentire una separazione delle cariche fotoprodotte. Questo è il prerequisito per ottenere dalla luce solare elettricità e i combustibili solari, come l'idrogeno dalla scissione fotocatalitica dell'acqua o sostanze organiche contenenti carbonio dalla riduzione del biossido di carbonio, quindi combustibili che possono essere immagazzinati e utilizzati al bisogno. La conversione dell'energia solare in energia chimica costituisce pertanto una grande sfida per la chimica.

$* * *$

ABSTRACT. - Energy is the most important issue of the $21^{\text {st }}$ century. Due to severe environmental problems, mainly related to air pollution of urban areas and green house effects leading to global warming, fossil fuels need to be replaced by renewable energy sources. Beside the wind energy, which is progressively more and more exploited, solar energy represents a clean, abundant, diffuse and economical energy which can be converted into heat, electricity or in the form of so called solar fuels, provided special materials are developed able to efficiently absorb solar light and produce charge separation. This is the prerequisite for obtaining electricity from sunlight and solar fuels, e.g.

(*) Dipartimento di Chimica, Università degli Studi di Milano, Italy.

E-mail: elena.selli@unimi.it 
hydrogen from water photosplitting or carbon containing species from carbon dioxide photoinduced reduction, which can be stored and used on need. Converting solar energy into chemical energy is thus a big challenge for chemistry.

I combustibili fossili hanno offerto enormi opportunità di sviluppo ai paesi del mondo occidentale durante il ventesimo secolo, ma già da tempo sono sorte grosse problematiche conseguenti al loro utilizzo. Non solo le riserve comprovate di combustibili fossili stanno progressivamente diminuendo e quindi occorre ricorrere in modo massiccio ad altre fonti di energia sostitutive, ma è ben evidente che il continuo utilizzo dei combustibili fossili comporta notevoli ricadute dannose, quali l'inquinamento ambientale che costituisce una minaccia per la salute e l'aumento dei gas serra con il conseguente riscaldamento globale del nostro pianeta e i disastrosi cambiamenti climatici che ne conseguono.

Senza dubbio l'energia costituisce il più importante problema del ventunesimo secolo [1]. Occorre trovare sorgenti di energia con cui sostituire i combustibili fossili e al tempo stesso far sì che tutti gli abitanti della terra possano raggiungere gli standard di vita delle nazioni sviluppate senza produrre devastazioni nel nostro pianeta, cercando quindi di lasciarlo in buono stato per le generazioni future. Tutti noi siamo quindi chiamati innanzitutto a evitare sprechi di energia e a contribuire allo sviluppo e al miglioramento delle tecnologie per l'energia perché il problema energetico possa essere risolto alla radice.

Probabilmente la conseguenza più pesante del consumo di combustibili fossili consiste nell'alterare il ciclo del carbonio che favorisce il riscaldamento globale. Anche se la quantità di carbonio immesso nell'aria a seguito dell'utilizzo dei combustibili fossili può sembrare piccolo in confronto con il suo continuo scambio che avviene naturalmente fra la biosfera e l'atmosfera, è tuttavia abbastanza, sul lungo termine, per far sì che la concentrazione di $\mathrm{CO}_{2}$ aumenti costantemente. Dalla rivoluzione industriale, in effetti, la concentrazione di $\mathrm{CO}_{2}$ nell'atmosfera è aumentata del $30 \%$. Ciò comporta un'alterazione dell'equilibrio di irraggiamento della terra e l'induzione di variazioni climatiche che si sovrappongono alle oscillazioni naturali [2], con il riscaldamento globale e lo scatenarsi di eventi climatici estremi, quali uragani, siccità o ondate di caldo. 


\section{COMBUSTIBILI FOSSILI}

Il petrolio è senza dubbio il combustibile ideale in quanto liquido e quindi facilmente estraibile e trasportabile. Dall'inizio del ventesimo secolo la domanda di petrolio è cresciuta costantemente negli anni ed il suo approvvigionamento è sempre stato in grado di far fronte alla domanda. Vi sarà un momento, tuttavia, in cui la fornitura di petrolio non sarà in grado di soddisfarne la domanda sempre crescente. In quel momento le riserve di petrolio non saranno esaurite, ma l'età d'oro del petrolio facile sarà finita.

Il picco nell'utilizzo del gas naturale, l'altra preziosa fonte di energia di origine fossile, è atteso più tardi rispetto al picco di utilizzo del petrolio. Tuttavia l'infrastruttura per la distribuzione del gas naturale è più rigida e fragile, rispetto a quella del petrolio. In linea di principio il problema della sua distribuzione può essere ridimensionato con l'utilizzo di navi a gas naturale liquefatto e di terminali costieri di rigassificazione, infrastrutture molto costose, che presentano gravi problemi di sicurezza.

Il carbone è il più abbondante, ma il più sporco dei combustibili fossili. Ma nei prossimi anni il problema del carbone non sarà tanto la sua disponibilità, ma la sua sostenibilità ambientale. L'uso dei combustibili che producono più gas serra è chiaramente in conflitto con gli attuali sforzi per una politica sul clima di scala mondiale.

La transizione già in atto dai combustibili fossili a qualche tipo di fonte di energia rinnovabile è quindi urgente e irrinunciabile. Né ci si può aspettare che la soluzione venga dall'utilizzo dell'energia nucleare. Infatti, oltre al problema, tuttora irrisolto, dello smaltimento sicuro delle scorie nucleari, che comporta la grossa preoccupazione di lasciare alle generazioni future depositi nucleari radioattivi per migliaia di anni, la possibilità concreta e comprovata del verificarsi di incidenti nelle centrali nucleari fa sì che ci si possa solo aspettare che il loro numero diminuirà, non aumenterà, negli anni futuri.

\section{FONTI DI ENERGIA RINNOVABILE}

Esistono forme di energia cosiddette rinnovabili che sono già competitive con l'utilizzo dei combustibili fossili, quali l'energia eolica, l'energia termoelettrica e forme di energia che utilizzano fenomeni locali. 
Produrre elettricità dal vento presenta una serie di notevoli vantaggi, quali, innanzitutto, il costo zero garantito del vento, l'assenza totale di emissioni nell'atmosfera e di calore da dissipare. Si tratta di una tecnologia relativamente semplice, con tempi di costruzione delle strutture relativamente brevi. Per questo il costo dell'elettricità prodotta per via eolica continuerà a diminuire sul lungo termine, rendendola sempre più competitiva rispetto ad altre tecnologie. Gli svantaggi sono correlati con la naturale variabilità dei venti, la distanza fra i campi eolici e i centri di consumo e le obiezioni di tipo estetico ed ecologico all'istallazione di pale eoliche. L'Europa ha un grosso potenziale di energia eolica, che in linea di principio dovrebbe poter soddisfare tutte le nostre necessità di elettricità.

L'energia idroelettrica è una delle tecnologie per la produzione di elettricità in uso da più tempo, per cui se ne possono facilmente valutare i pro e contro. Fra questi ultimi, per prima l'invasività. Nel ventesimo secolo la costruzione di grandi dighe ha comportato la necessita di dislocamento di popolazioni soprattutto nei paesi in via di sviluppo, con incalcolabili ricadute di tipo sociale ed economico. Sono stati inondati ecosistemi naturali unici, con conseguenti cambiamenti drammatici della vita nei fiumi a valle delle dighe. Nonostante questo, la produzione di energia idroelettrica presenta innegabili vantaggi, fra cui i bassi costi operativi e la durata più lunga degli impianti rispetto a qualsiasi altro modo di produzione di elettricità, il facile accumulo di energia potenziale che consente di far fronte alla domanda di picchi di elettricità, la possibilità di fornire acqua da bere e per irrigare, la prevenzione di inondazioni ricorrenti, talora distruttive. Enorme è in linea di principio il potenziale idroelettrico non sfruttato della terra; tuttavia soltanto una sua frazione molto limitata può essere utilizzata in modo sostenibile dal punto di vista economico e ambientale. Per quanto riguarda l'Europa, il potenziale per nuovi impianti idroelettrici è minimo, ma sistemi di piccole dimensioni largamente distribuiti sul territorio potranno in futuro dare un significativo contributo al fabbisogno di energia.

Fra le altre opzioni che madre natura ci regala per trarre energia dalla terra, la più importante è probabilmente l'energia geotermica derivante dal calore immagazzinato in profondità del nostro pianeta, che può essere estratta ed usata direttamente come calore o convertita in elettricità. Come sorgenti di energia possono essere utilizzati anche i 
gradienti di temperatura di oceani e laghi, le correnti e le onde, mentre l'energia gravitazionale terra-luna può essere sfruttata in zone costiere dell'emisfero nord dove le maree muovono grossi volumi di acqua di mare in un tempo relativamente breve.

\section{L'ENERGIA SOLARE}

La vita sulla terra non sarebbe possibile senza l'irraggiamento solare, che ci garantisce un flusso di energia inesauribile, in quantità che supera di gran lunga le necessità dell'umanità. Il sole è la stella madre del sistema solare (Fig. 1). È una nana gialla, la cui superficie è costituita da idrogeno $(74 \%)$ ed elio $(25 \%)$, con tracce di altri elementi più pesanti.

L'energia solare rende possibile la vita sul nostro pianeta. Senza la luce solare, infatti, non sarebbe possibile la fotosintesi, attraverso cui la $\mathrm{CO}_{2}$ viene trasformata in composti organici e l'ossigeno ha raggiunto l'attuale concentrazione nell'atmosfera terrestre.

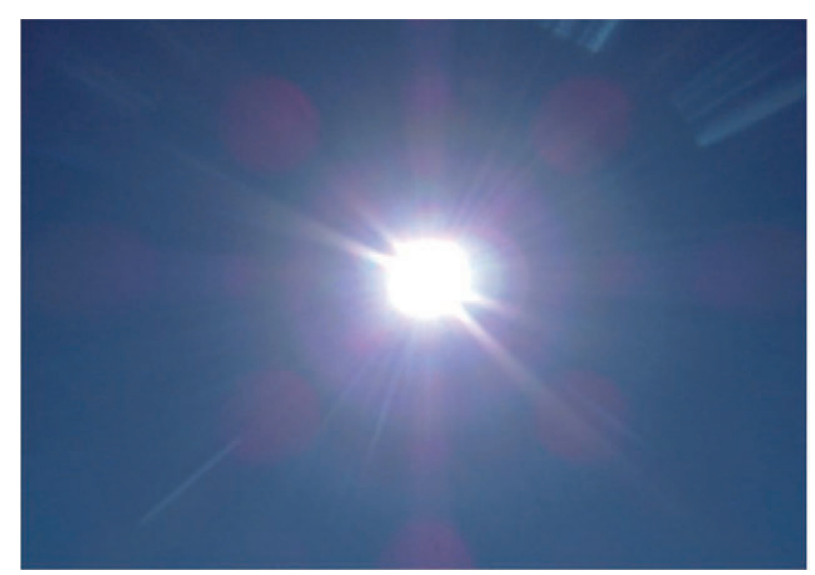

Fig. 1. Il Sole.

L'importanza del sole per la vita sulla terra è ben riconosciuto fin dall'antichità. In molte culture antiche e preistoriche, ad esempio quelle degli Inca e degli Aztechi, il sole era concepito come una divinità. Nella religione egizia il Sole era la divinità più importante e il faraone, considerato una divinità in terra, era ritenuto il figlio del Sole. 
Antichi monumenti furono costruiti tenendo conto della posizione del sole nei vari periodi dell'anno: ad esempio i megaliti di Stonehenge (Fig. 2) segnano il solstizio d'estate. Nella mitologia greca la divinità solare principale fu Helios, in epoca storica confuso con Apollo, rappresentato alla guida del carro del sole, una quadriga tirata da cavalli che soffiano fuoco dalle narici (Fig. 3).
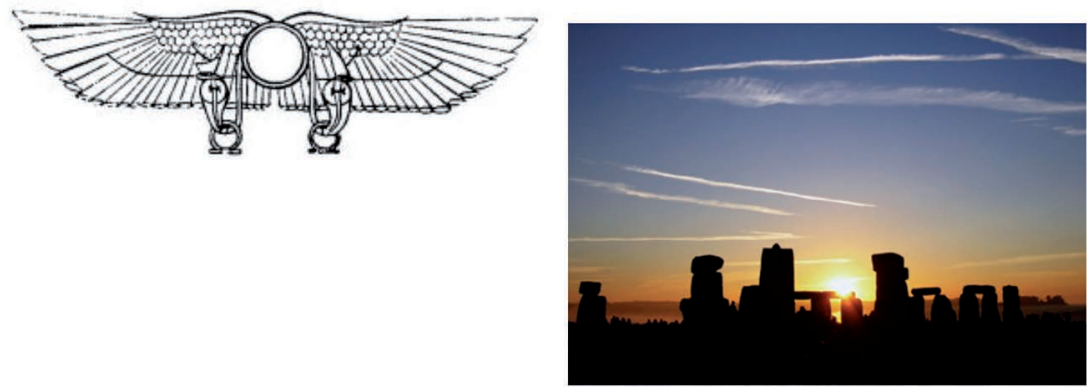

Fig. 2. I megaliti di Stonehenge.
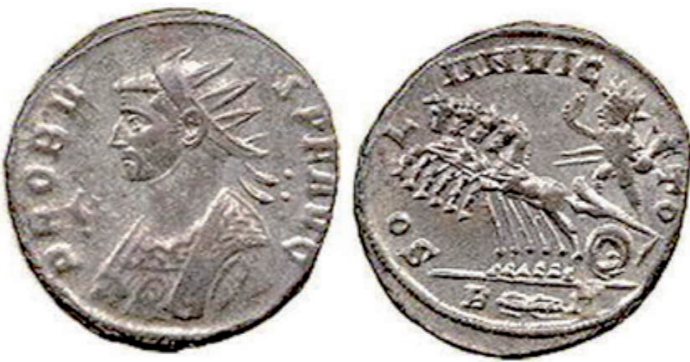

Fig. 3. Monete antiche.

L'energia solare ha un enorme potenziale come fonte di energia pulita, abbondante ed economica, che, tuttavia, non può essere adoperata come tale, ma deve essere raccolta e convertita in altre forme di energia facilmente utilizzabili. Poiché l'energia solare è diffusa e intermittente, la sua conversione deve comportare la sua concentrazione ed il suo immagazzinamento. Ma attualmente nessuna delle vie utilizzate per convertire l'energia solare in calore, elettricità o combustibili è competitiva rispetto all'utilizzo dei combustibili fossili al loro prezzo attuale di mercato. 
Il solare termico. Il modo con cui l'energia solare viene al momento maggiormente usata consiste nell'utilizzo dei collettori termici solari, in cui un liquido viene scaldato sotto irraggiamento solare per fornire acqua calda per uso diretto o per riscaldamento domestico (Fig. 4). Si tratta di sistemi molto semplici, che non richiedono la concentrazione della luce solare. Questo modo di utilizzo dell'energia solare potrà avere un ruolo molto rilevante, se si considera che nelle case moderne più del $50 \%$ dell'energia utilizzata viene consumata banalmente per scaldare l'acqua per riscaldarsi, lavarsi e cucinare. Quindi una larga diffusione del solare termico comporterà un sostanziale alleggerimento del conto dell'energia nel settore residenziale e delle relative conseguenze ambientali.

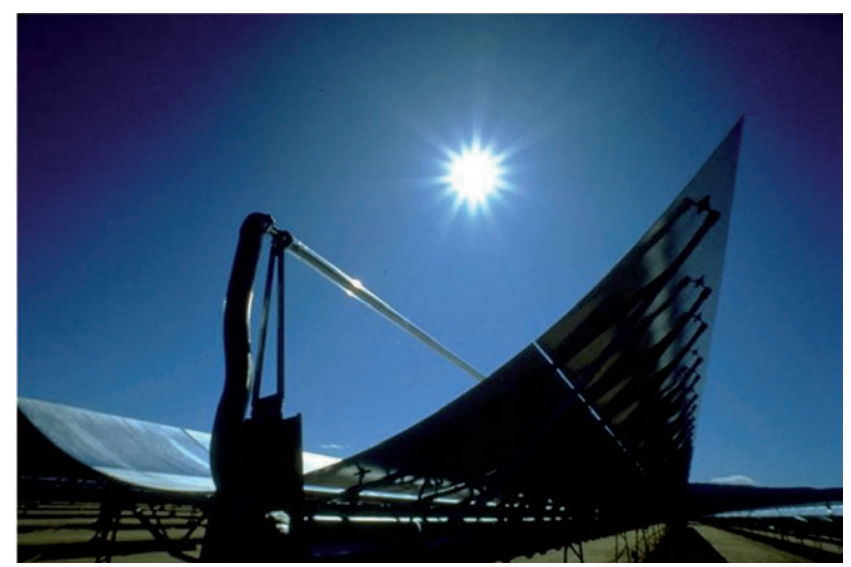

Fig. 4. Collettori solari.

L'energia solare può anche essere convertita in calore ad alta temperatura usando specchi che concentrano la radiazione solare (Fig. 5) e quindi in elettricità, o usata direttamente in processi termochimici.

La concentrazione di luce solare è stata usata fin dai tempi dell'antica Cina (Fig. 6). Si tramanda anche che Archimede usò scudi ben lucidati come specchi per concentrare la luce del sole sulla flotta romana che stava assaltando Siracusa nel 212 A.C.

L'elettricità solare. L'elettricità può essere prodotta dalla luce solare mediante celle fotovoltaiche e dispositivi correlati, oltre che da siste$\mathrm{mi}$ ad alta temperatura. Le celle fotovoltaiche costituiscono il sistema di gran lunga più promettente per la produzione di elettricità diffusa, 
hanno molte applicazioni e sono particolarmente adatte a situazioni in cui non si ha a disposizione l'energia elettrica della rete. D'altra parte nei paesi sviluppati le celle solari, in forma di moduli o di pannelli solari sui tetti degli edifici, possono essere collegate alla rete di distribuzione dell'energia elettrica.

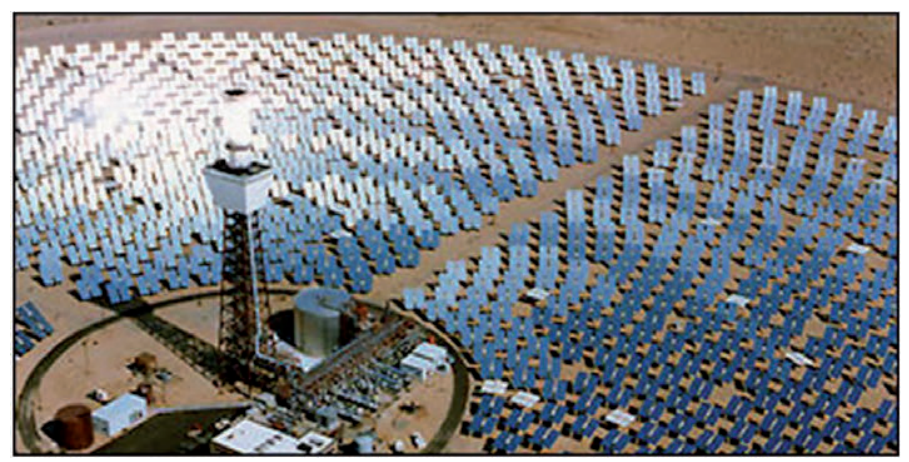

Fig. 5. Il sistema di concentrazione della luce solare alla Plataforma Solar de Almeria.

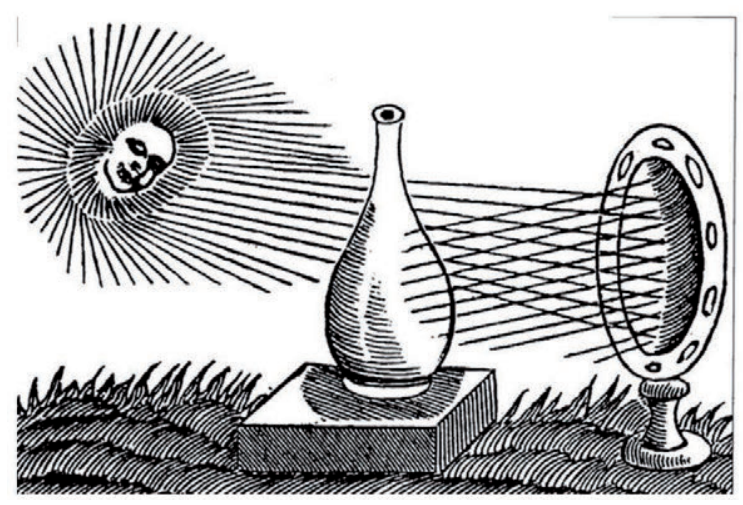

Fig. 6. Concentrazione di luce solare mediante specchio.

Il costo dell'elettricità prodotta dalla luce solare è già competitivo con quello dell'elettricità prodotta da sorgenti fossili e dal nucleare. In questo campo continua un'intensa e estesa ricerca per produrre nuovi materiali più performanti in grado di assorbire la luce solare e migliorare la tecnologia, per aumentare l'efficienza delle celle fotovoltaiche e ridurne i costi. 
L'energia solare può essere convertita in energia elettrica anche in celle fotoelettrochimiche basate su elettrodi a semiconduttore in contatto con un elettrolita che consente scambio di elettroni (Fig. 7). Il semiconduttore più stabile e quindi di gran lunga più utilizzato fino a poco fa è il biossido di titanio, $\mathrm{TiO}_{2}$, che tuttavia è in grado di assorbire solo una frazione relativamente piccola della luce solare perché il suo band gap è troppo largo $(3.0 \mathrm{eV})$. Per superare questo problema, la luce può essere fatta assorbire da molecole di colorante adsorbite su film sottili di particelle nanocristalline di $\mathrm{TiO}_{2}$ [3]. Le molecole di colorante, elettronicamente eccitate a seguito dell'assorbimento di luce solare, iniettano elettroni nel $\mathrm{TiO}_{2}$, producendo processi di separazione di carica. Le celle solari sensibilizzate da coloranti, le cosiddette $d y e$ sensitized solar cells, sono interessanti per il basso costo del $\mathrm{TiO}_{2}$ e per la potenziale semplicità del loro processo di costruzione. Tuttavia non hanno rese di conversione elevate e presentano problemi principalmente legati alla stabilità dei coloranti. Negli ultimi anni sono state superate da celle basate su materiali diversi, le perovskiti, con le quali posso essere ottenute rese di conversione molto maggiori, pur presentando problemi, soprattutto di stabilità, ancora non risolti.

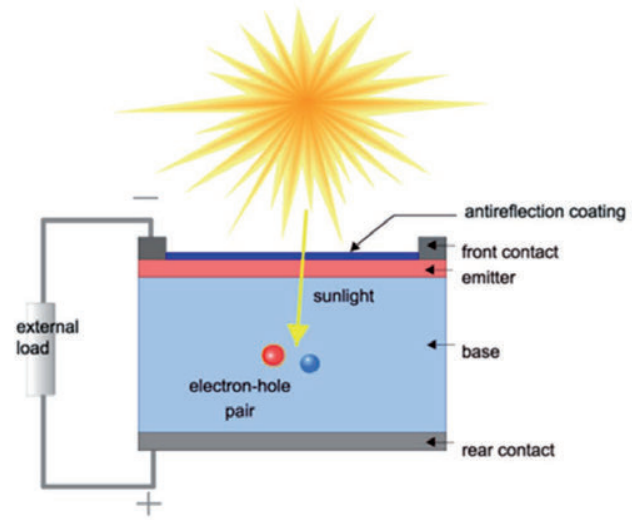

Fig. 7. Cella fotoelettrochimica.

Combustibili solari e fotosintesi artificiale. I combustibili liquidi rappresentano la miglior forma di energia in quanto possono essere facilmente conservati e trasportati. A causa della natura intermittente della luce solare, la sua conversione in combustibili chimici facilmente 
immagazzinabili e trasportabili attraverso un processo analogo a quello che avviene nella fotosintesi naturale costituisce sicuramente la soluzione di gran lunga più vantaggiosa per l'utilizzo dell'energia solare.

La fotosintesi naturale, infatti, converte la luce solare in combustibili, producendo biomassa, che in tempi geologici si è poi trasformata in combustibili fossili. Tuttavia la velocità di produzione di combustibili fossili è 500.000 volte inferiore rispetto alla velocità con cui vengono attualmente consumati. Globalmente, solo lo $0.3 \%$ dell'energia solare che raggiunge la terra viene immagazzinata sotto forma di materia delle piante [1]. Aumentare l'efficienza della fotosintesi naturale sarebbe ovviamente un risultato importante, per raggiungere il quale è essenziale chiarire le basi molecolari del processo di fotosintesi naturale. Negli organismi fotosintetici la luce viene raccolta da sistemi antenna che consistono in complessi pigmento-proteina. L'energia di eccitazione così catturata viene poi trasferita ai centri di reazione dove viene convertita in energia potenziale elettrochimica in un processo di trasferimento elettronico allo stato eccitato. Gli equivalenti ossidanti e riducenti che ne derivano sono quindi trasportati ai siti catalitici, attraverso successivi step di trasferimento elettronico, e qui sono utilizzati per ossidare l'acqua e produrre combustibili, quali i carboidrati.

La necessità e la possibilità di ottenere la fotosintesi artificiale [4] è stata anticipata più di un secolo fa dal chimico italiano Giacomo Ciamician (Fig. 8), che in un famoso articolo [5] pubblicato su Science nel 1912 scrisse quanto segue:

When vegetation is rich, photochemistry may be left to the plants and, by rational cultivation, solar radiation may be used for industrial purposes. In the desert regions, unsuitable for any kind of cultivation, photochemistry will artificially put their solar energy to practical uses. On the arid lands there will spring up industrial colonies without smoke and without smokestacks; forests of glass tubes will extend over the plants and glass buildings will rise everywhere; inside of these will take place the photochemical processes that hitherto have been the guarded secret of the plants, but that will have been mastered by buman industry which will know how to make them bear even more abundant fruit than nature, for nature is not in a burry and mankind is. And if in a distant future the supply of coal becomes completely exhausted, civilization will not be checked by that, for life and civilization will continue as long as the sun shines! 


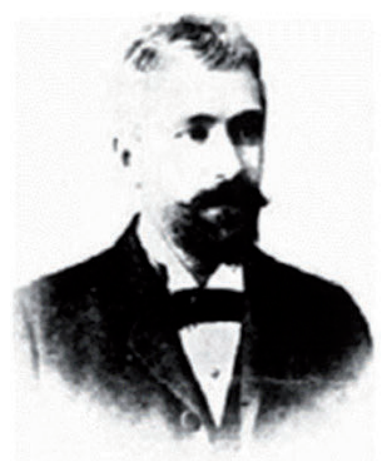

Fig. 8. Giacomo Ciamician.

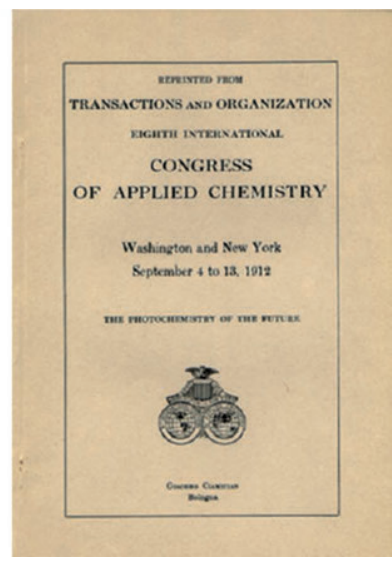

La produzione efficiente di combustibili solari puliti rappresenta senza dubbio una delle più importanti sfide della scienza moderna. Perché la produzione di combustibili solari sia vantaggiosa dal punto di vista economico e ambientale, occorre ottenerli a partire da materiali di partenza abbondanti e non costosi, quali l'acqua e il biossido di carbonio. L'acqua può essere scissa in idrogeno e in ossigeno molecolari sulla superficie di un semiconduttore in grado di assorbire la luce solare e produrre una efficiente separazione di carica (Fig. 9). E il biossido di carbonio in soluzione acquosa può essere ridotto, ad esempio, a etanolo con la concomitante generazione di ossigeno molecolare.

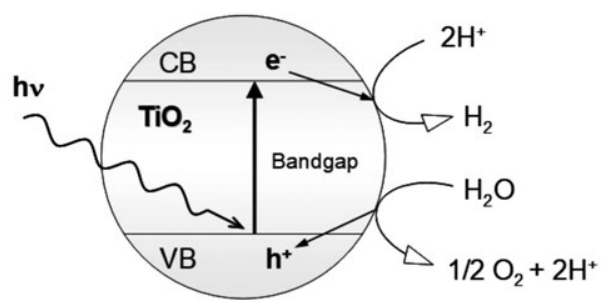

Fig. 9. Scissione fotocatalitica dell'acqua in idrogeno e ossigeno su biossido di titanio.

Su queste due semplici reazioni, e in particolare sullo sviluppo di materiali fotoattivi stabili in grado di assorbire in maniera efficiente la luce solare e consentire una buona separazione delle cariche fotogenera- 
te, si è principalmente focalizzata negli ultimi anni la ricerca sulla conversione di energia solare in energia chimica. L'idrogeno molecolare $\mathrm{H}_{2}$ fotogenerato per scissione fotocatalitica dell'acqua è il combustibile pulito per eccellenza, in quanto la sua combustione con ossigeno produce calore e acqua e la sua combinazione con ossigeno in una fuel cell genera elettricità, calore e acqua, senza alcuna immissione di $\mathrm{CO}_{2}$ nell'atmosfera.

Il modo migliore per costruire sistemi fotosintetici artificiali per la produzione di combustibili solari consiste nel cercare di imitare l'organizzazione molecolare e supramolecolare del processo fotosintetico naturale: la raccolta di luce deve portare ad una separazione di carica, seguita da un trasporto di carica per trasferire gli equivalenti ossidanti e riducenti ai siti catalitici dove avviene separatamente lo sviluppo di ossigeno e lo sviluppo di idrogeno (o la riduzione di $\mathrm{CO}_{2}$ ). Progressi sono indubbiamente stati fatti sui singoli aspetti della fotosintesi artificiale, con l'obiettivo di raggiungere l'integrazione dei vari componenti in un sistema attivo.

Il problema principale consiste nel fatto che la formazione di idrogeno e di ossigeno dall'acqua (così come la riduzione di $\mathrm{CO}_{2}$ ) sono processi a molti elettroni, mentre l'assorbimento di luce è un processo a un fotone che comporta un processo di separazione di carica a un elettrone. Sono quindi necessari catalizzatori che accoppino eventi a singolo elettrone in processi in grado di accumulare gli equivalenti redox necessari per la produzione di carburanti. La ricerca è particolarmente focalizzata sullo sviluppo di catalizzatori efficienti per la generazione di ossigeno, che è un processo che implica il trasferimento di quattro elettroni.

Combustibili da biomassa. Occorre infine ricordare che la biomassa stessa, prodotta dalla fotosintesi naturale, è stata e continua ad essere un'importante risorsa per la produzione di energia, in particolare nei paesi in via di sviluppo, dove è spesso utilizzata per fornire energia in industrie su piccola scala e più in generale come legno combustibile da bruciare nelle stufe. Questo può comportare grossi problemi respiratori dovuti all'inquinamento dell'aria interna in conseguenza della cattiva ventilazione delle stufe alimentate con biomassa.

Nei paesi industrializzati la biomassa è utilizzata per produrre calore ed elettricità usando principalmente biomassa solida, o per ottenere combustibili liquidi, quali il bioetanolo e il biodiesel. L'etanolo viene estratto dalla canna da zucchero in Brasile e dal mais negli Stati Uniti. Una estensiva produzione di energia da biomassa richiede ovviamente aree di terra coltivabile molto grandi ed enormi quantità di acqua. 


\section{SVILUPPO DI MATERIALI E DISPOSITIVI PER LA CONVERSIONE DI ENERGIA SOLARE}

Il gruppo di ricerca da me diretto nel Dipartimento di Chimica dell'Università degli Studi di Milano si dedica da anni alla conversione di energia solare in energia chimica, in particolare allo sviluppo di materiali fotocatalitici in grado di assorbire la luce solare e produrre idrogeno da soluzioni acquose, ovvero migliorare la separazione delle cariche prodotte nel materiale a seguito dell'assorbimento di luce, al fine di costruire fotoanodi in celle fotoeletrocatalitiche. Tali materiali sono stati utilizzati anche nella costruzione di celle e devices per la produzione di corrente e lo sviluppo separato di idrogeno e ossigeno mediante scissione fotocatalitica dell'acqua.

Come già accennato, il biossido di titanio è il materiale semiconduttore fin qui più largamente usato in fotocatalisi e fotoelettrocatalisi, sostanzialmente perché poco costoso e stabile anche sotto irraggiamento. Il principale limite nell'utilizzo del $\mathrm{TiO}_{2}$ come materiale fotoattivo consiste nel fatto che è in grado di assorbire solo una piccola porzione della luce emessa dal sole. Pertanto sono stati studiati materiali a base di $\mathrm{TiO}_{2}$ che assorbono una porzione più larga dello spettro solare e che consentono anche una migliore separazione delle cariche fotogenerate in tale materiale. Uno dei modi di spostare l'assorbimento del materiale a lunghezze d'onda più lunghe consiste nell'introduzione di stati all'interno del band gap del semiconduttore mediante drogaggio del materiale, ad esempio sostituendo parzialmente l'ossigeno nel $\mathrm{TiO}_{2}$ con elementi quali carbonio o azoto [6]. Tuttavia in questo modo la mobilità delle cariche fotogenerate nel materiale per assorbimento di radiazione risulta molto limitata e i fotocatalizzatori ottenuti, pur assorbendo una frazione maggiore della luce solare, risultano meno efficienti.

Risultati molto interessanti sono stati invece ottenuti mediante fluorurazione del $\mathrm{TiO}_{2}$ [6]. Mediante studi di fluorescenza risolta nel tempo, abbiamo dimostrato che in tali materiali si generano difetti superficiali che fungono da trappole per le lacune elettroniche fotogenerate, con effetti modesti sulle proprietà di assorbimento [7], ma con una migliore separazione di carica fotoindotta e conseguente aumento dell'attività fotocatalitica del materiale [8].

Per lo sviluppo fotocatalitico di idrogeno da soluzioni acquose particolarmente efficienti sono materiali a base di $\mathrm{TiO}_{2}$ modificati per 
deposizione di nanoparticelle di metalli [9], sia nobili (NM), quali oro [10-12] e soprattutto platino [13], che rame [14], preparati con un metodo innovativo di flame spray pyrolysis [11-13] sviluppato nei nostri laboratori (Fig. 10). L'efficienza di tali materiali risulta molto più elevata $[15,16]$, principalmente perché le nanoparticelle di NM sono in grado di catturare gli elettroni fotopromossi nella banda di conduzione del semiconduttore e facilitare quindi la riduzione di acqua a idrogeno (Fig. 11). Tali materiali sono stati studiati principalmente nella reazione di produzione fotocatalitica di idrogeno da soluzioni acquose, ma ci siamo interessati anche della riduzione fotocatalitica di $\mathrm{CO}_{2}[17,18]$.

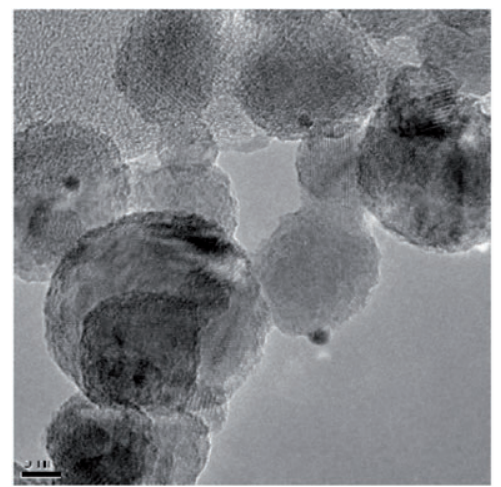

Fig. 10. Particelle di biossido di titanio contenenti nanoparticelle di metallo, ottenute per flame spray pyrolysis.

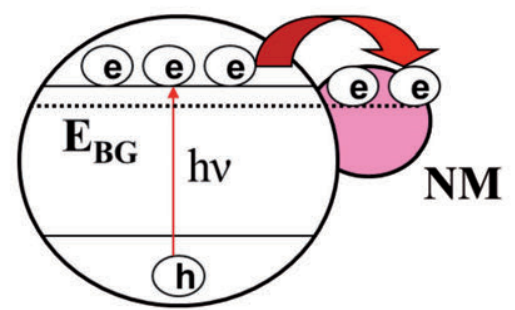

Fig. 11. Efficiente separazione di carica in semiconduttori modificati con nanoparticelle di metalli nobili (NM).

Recente è lo studio delle eterogiunzioni fra bismuto vanadato e triossido di tungsteno per la fabbricazione di fotoanodi per celle solari (Fig. 12), in cui l'efficienza di conversione in corrente della luce a diverse lunghezze d'onda è stata razionalizzata sulla base della dinamica 
ultraveloce dei transienti fotogenerati nel materiale, studiata mediante spettroscopia di assorbimento al femtosecondo [19,20].

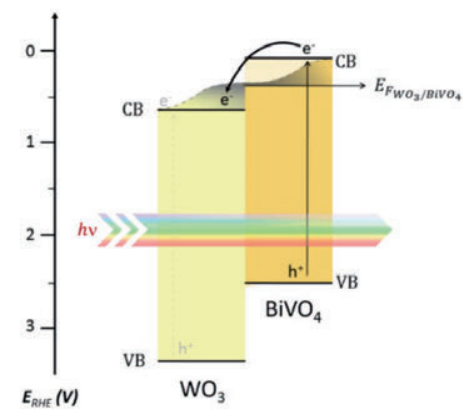

Fig. 12. Eterogiunzione bismuto vanadato-triossido di tungsteno per la fabbricazione di fotoanodi.

Sono stati infine sviluppati dispositivi per la scissione fotocatalica dell'acqua, a partire da una cella a due scomparti per l'evoluzione separata di idrogeno e ossigeno da soluzioni acquose (Fig. 13). L'elemento fondamentale di tale cella è costituito da un elettrodo di titanio ricoperto da un lato di $\mathrm{TiO}_{2}$, che funge da fotoanodo, mentre gli elettroni fotopromossi nel sistema migrano attraverso l'elettrodo all'altro scomparto della cella dove avviene lo sviluppo di idrogeno per riduzione dei protoni dell'acqua [21]. I più recenti sviluppi di tale elettrodo consistono nell'utilizzo di $\mathrm{TiO}_{2}$ in forma di nanotubi ottenuti per anodizzazione di una lastra di titanio [22], che hanno mostrato anche interessanti proprietà di cristalli fotonici [23] (Fig. 14).
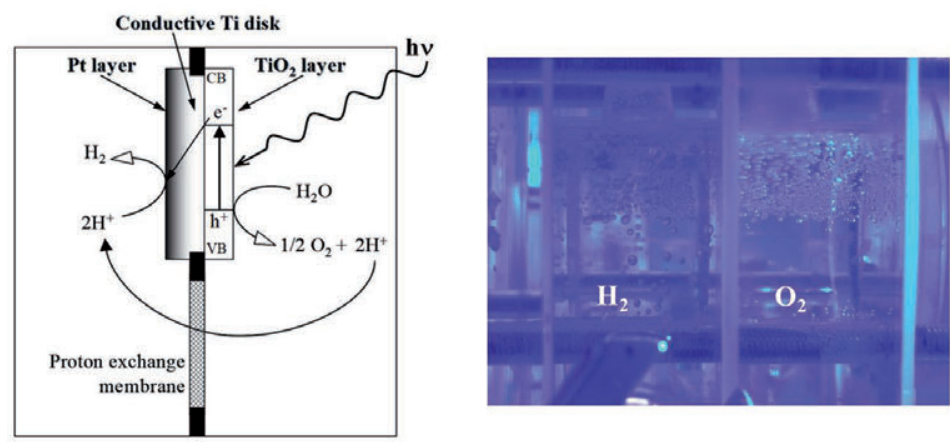

Fig. 13. Fotoelettrodo della cella a scomparti separati e immagine della cella stessa. 

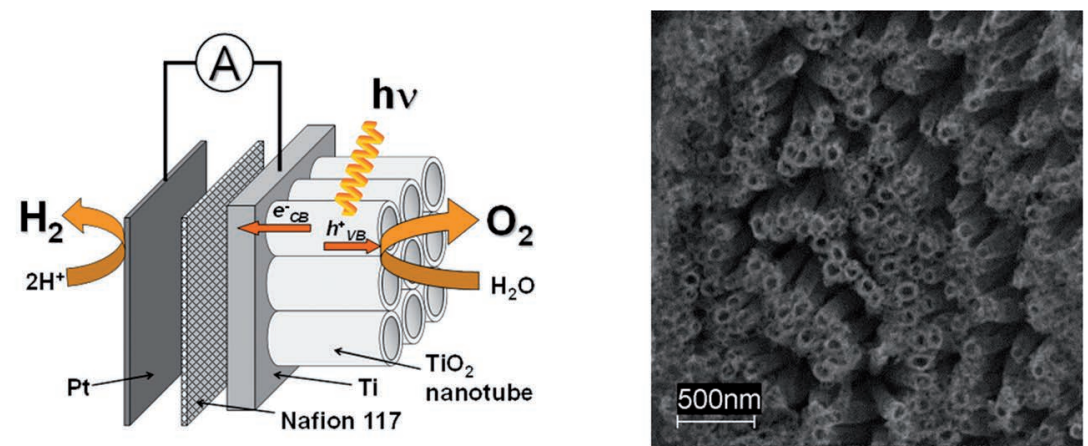

Fig. 14. Cella fotoelettrocatalitica per la scissione dell'acqua in idrogeno e ossigeno e simultanea misura della fotocorrente generata. Il materiale fotocatalitico è costituito da nanotubi di $\mathrm{TiO}_{2}$ (immagine a destra) cresciuti per anodizzazione di una lastra sottile di titanio.

\section{CONCLUSIONI}

L'energia solare è abbondante e inesauribile, è diffusa su tutta la terra e può essere trasformata in altre forme di energia utilizzabili dall'uomo attraverso una serie di tecnologie, anche relativamente semplici, che non implicano la possibilità di gravi incidenti, né un cattivo uso per scopi militari, ma che necessitano di ulteriori sviluppi per divenire competitive rispetto all'utilizzo dei combustibili fossili.

La chimica ha contribuito e sta contribuendo largamente a risolvere il problema energia principalmente giocando un ruolo chiave nello sviluppo delle tecnologie correlata con le tre strade di conversione dell'energia solare (calore solare, elettricità solare, combustibili solari), due delle quali implicano processi chimici, che richiedono lo sviluppo di materiali speciali. Le performances dei sistemi di assorbimento di luce, conversione e immagazzinamento di energia potranno essere largamente migliorate attraverso la progettazione razionale e l'applicazione sperimentale di nuovi materiali e processi. Ci stiamo lavorando in molti. Trovare una o più vie di uscita per risolvere il problema energetico e mitigare i problemi ambientali correlati con l'uso di combustibili fossili è una grande sfida per la chimica. 


\section{RINGRAZIAMENTI}

I risultati delle ricerche sui materiali fotocatalitici qui brevemente presentati sono stati ottenuti in parte nell'ambito del progetto congiunto Regione Lombardia-Fondazione Cariplo SmartMatLab, di cui sono stata Principal Investigator, nonché grazie ad altri progetti di ricerca su materiali e dispositivi foto(elettro)catalitici finanziati da Fondazione Cariplo e dal Ministero dell'Università e della Ricerca (progetti PRIN).

\section{BIBLIOGRAFIA}

1. Armaroli N, Balzani V, The Future of Energy Supply: Challenges and Opportunities, Angew. Chem. Int. Ed., 2007: 46: 52-66.

2. Kerr RA, A worrying trend of less ice, higher seas, Science, 2006: 311: 1698-1701.

3. Grätzel M, Solar energy conversion by dye-sensitized photovoltaic cells, Inorg. Chem., 2005: 44: 6841-6851.

4. Collings A, Critchley C, Artificial Photosynthesis, Wiley-VCH, Weinheim, 2005.

5. Ciamician G, The photochemistry of the future, Science, 1912: 36: 385-394.

6. Dozzi MV, Selli E, Doping $\mathrm{TiO}_{2}$ with p-Block Elements: Effects on Photocatalytic Activity, J. Photochem. Photobiol. C. Photochem. Rev., 2013: 14: 13-28.

7. Dozzi MV, Ohtani B, Selli E, Absorption and Action Spectra Analysis of Ammonium Fluoride-doped Titania Photocatalysts, Phys. Chem. Chem. Phys., 2011: 13: 18217-18227.

8. Dozzi MV, D'Andrea C, Ohtani B, Valentini G, Selli E, Fluorine-doped $\mathrm{TiO}_{2}$ materials: photocatalytic activity vs. time-resolved photoluminescence, J. Phys. Chem. C, 2013: 117: 25586-25595.

9. Chiarello GL, Dozzi MV, Selli E, $\mathrm{TiO}_{2}$-based materials for photocatalytic hydrogen production, J. Energy Chem., 2017: 26: 250-258.

10. Chiarello GL, Selli E, Forni L, Photocatalytic hydrogen production over flame spray pyrolysis-synthesised $\mathrm{TiO}_{2}$ and $\mathrm{Au} / \mathrm{TiO}_{2}$, Appl. Catal. B: Environ., 2008, 84: 332-339.

11. Chiarello GL, Forni L, Selli E, Photocatalytic hydrogen production by liquidand gas-phase reforming of $\mathrm{CH}_{3} \mathrm{OH}$ over flame-made $\mathrm{TiO}_{2}$ and $\mathrm{Au} / \mathrm{TiO}_{2}$, Catal. Today, 2009: 144: 69-74.

12. Dozzi MV, Prati L, Canton P, Selli E, Effects of gold nanoparticles deposition on the photocatalytic activity of titanium dioxide under visible light, Phys. Chem. Chem. Phys., 2009: 11: 7171-7180.

13. Chiarello GL, Dozzi MV, Scavini M, Grunwaldt JD, Selli E, One step flamemade fluorinated $\mathrm{Pt} / \mathrm{TiO}_{2}$ photocatalysts for hydrogen production, Appl. Catal. B: Environ., 2014: 160-161: 144-151.

14. Dozzi MV, Chiarello GL, Pedroni M, Livraghi S, Giamello E, Selli E, High photocatalytic hydrogen production on $\mathrm{Cu}(\mathrm{II})$ pre-grafted $\mathrm{Pt} / \mathrm{TiO}_{2}$, Appl. Catal. B: Environ., 2017: 209: 417-428. 
15. Chiarello GL, Aguirre MH, Selli E, Hydrogen production by photocatalytic steam reforming of methanol on noble metal modified $\mathrm{TiO}_{2}$, J. Catal., 2010: 273: 182-190.

16. Chiarello GL, Ferri D, Selli E, Effect of the $\mathrm{CH}_{3} \mathrm{OH} / \mathrm{H}_{2} \mathrm{O}$ ratio on the mechanism of the gas-phase photocatalytic reforming of methanol on noble metalmodified $\mathrm{TiO}_{2}$, J. Catal., 2011: 280: 168-177.

17. Riboni F, Selli E, Hoffmann MR, Colussi AJ, Homogeneous Reduction of $\mathrm{CO}_{2}$ by Photogenerated Pyridinyl Radicals, J. Phys. Chem. A, 2015: 119: 4433-4438.

18. Grigioni I, Dozzi MV, Bernareggi M, Chiarello GL, Selli E, Photocatalytic $\mathrm{CO}_{2}$ reduction vs. $\mathrm{H}_{2}$ production: the effects of surface carbon-containing impurities on the performance of $\mathrm{TiO}_{2}$-based photocatalysts, Catal. Today, 2017: 281: 214-220.

19. Grigioni I, Stamplecoskie KG, Selli E, Kamat PV, Dynamics of Photogenerated Charge Carriers in $\mathrm{WO}_{3} / \mathrm{BiVO}_{4}$ Heterojunction Photoanodes, J. Phys. Chem. C, 2015: 119: 20792-20800.

20. Grigioni I, Stamplecoskie KG, Jara DH, Dozzi MV, Oriana A, Cerullo G, Kamat PV, Selli E, Wavelength-Dependent Ultrafast Charge Carrier Separation in the $\mathrm{WO}_{3} / \mathrm{BiVO}_{4}$ Coupled System, ACS Energy Lett., 2017: 2: 1362-1367.

21. Selli E, Chiarello GL, Quartarone E, Mustarelli P, Rossetti I, Forni L, A photocatalytic water splitting device for separate hydrogen and oxygen evolution, Chem. Commun., 2007: 5022-5024.

22. Altomare M, Pozzi M, Allieta M, Bettini LG, Selli E, $\mathrm{H}_{2}$ and $\mathrm{O}_{2}$ photocatalytic production on $\mathrm{TiO}_{2}$ nanotube arrays: effect of the anodization time on structural features and photoactivity, Appl. Catal. B: Environ., 2013: 136-137: 81-88.

23. Yoo, JE, Lee K, Altomare M, Selli E, Schmuki P, Self-Organized Arrays of Single-Metal Catalyst Particles-in- $\mathrm{TiO}_{2}$ Cavities: A Highly Efficient Photocatalytic System, Angew. Chem., Int. Ed., 2013: 52: 7514-7517. 